\title{
The LHCb trigger in Run II
}

\author{
Emanuele Michielin*t \\ University and INFN of Padova \\ E-mail: emanuele.michielin@cern.ch
}

The LHCb trigger system has been upgraded to allow alignment, calibration and physics analysis to be performed in real time. An increased CPU capacity and improvements in the software have allowed lifetime unbiased selections of beauty and charm decays in the high level trigger. Thanks to offline quality event reconstruction already available online, physics analyses can be performed directly on this information and for the majority of charm physics selections a reduced event format can be written out. Beauty hadron decays are more efficiently triggered by re-optimised inclusive selections, and the HLT2 output event rate is increased by a factor of three.

38th International Conference on High Energy Physics 3-10 August 2016

Chicago, USA

*Speaker.

${ }^{\dagger}$ On behalf of the LHCb collaboration. 


\section{The $\mathrm{LHCb}$ detector}

The LHCb detector [1] is a single-arm forward spectrometer, covering the pseudorapidity range $2<\eta<5$ and designed for the study of particles containing b or c quarks. In Run I (20102012) of the LHC, the LHCb experiment collected a total luminosity of $3 \mathrm{fb}^{-1}$ of pp collisions at 7 and $8 \mathrm{TeV}$ centre-of-mass energies. Run-II of the LHC started in 2015 with a centre-of-mass energy of $13 \mathrm{TeV}$ and with a higher bunch crossing rate. Run II will continue until 2018, allowing LHCb to collect about $5 \mathrm{fb}^{-1}$ of data.

To perform precise heavy flavor physics measurements, the detector includes a high-precision tracking system which provides a measurement of momentum of charged particles with a relative uncertainty that varies from $0.5 \%$ at low momentum to $1.0 \%$ at $200 \mathrm{GeV} / \mathrm{c}$. This tracking system consists of a silicon-strip vertex detector (VELO) surrounding the proton-proton (pp) interaction region, a large-area silicon-strip detector located upstream of a dipole magnet with a bending power of about $4 \mathrm{Tm}$, and three $\mathrm{T}$ stations of silicon-strip detectors and straw drift tubes placed downstream of the magnet.

Different types of charged hadrons are distinguished using information from two ring-imaging Cherenkov detectors (RICHs). Photons, electrons and hadrons are identified by a calorimeter system consisting of scintillating-pad and preshower detectors, an electromagnetic calorimeter and a hadronic calorimeter. Muons are identified by a system composed of alternating layers of iron and multiwire proportional chambers.

Due to the high collision rate provided by the LHC only a fraction of events reconstructed in LHCb can be retained. The task of deciding which events to keep or discard is handled by the trigger system. The LHCb trigger is organized in three levels: the hardware Level-0 (L0) trigger and the software High Level Triggers HLT1 and HLT2. The software trigger has been redesigned during the long shutdown to allow the most possible wide and precise physics program during the Run II.

\section{The High Level Trigger in Run II}

Figure 1 shows the comparison between the Run I and the Run II trigger scheme. In Run I the output rate of events written to permanent offline storage was $5 \mathrm{kHz}$. Almost all events accepted by the HLT were recorded in a format containing all raw information from the detector. In order to be performed at the full bunch crossing frequency in real time, the event reconstruction performed in the trigger was simpler than the one used offline. The detector calibration and alignment parameters were obtained offline on triggered data afterward, when all the events were reconstructed offline to achieve the best performance regardless of the timing required. This strategy makes inefficient use of the computing resources and could also cause difficulties in understanding efficiencies due to the differences between online and offline reconstructions.

For Run II, a new trigger scheme has been developed to cope with the almost double visible rate, see Figure 1 on the right. The major change to the trigger strategy is that the processing of the second step of the software trigger (HLT2) has been completely deferred, in order to optimize the usage of the event filter farm (EFF) [2] where the software trigger is run. The EFF consists of approximately 1700 nodes with 27000 physical cores; 800 of which newly installed for Run II. The 
EFF hosts 5 PB of disk space which is exploited as a buffer, distributed in such a way that farm nodes with faster processors get a larger portion of the disk buffer. At an average event size of 55 kB passing HLT1, this buffer allows for approximately 150 hours of HLT1 datataking before HLT2 has to be executed.

The increased computing power in the EFF allows to perform low transverse momentum tracking without impact parameter cuts in HLT1 and the possibility to perform full offline quality reconstruction upfront in HLT2. The first has led to the development, for the first time at a hadronic collider experiment, of a set of HLT1 lines without any lifetime biasing cuts [3]. Physics analyses which can particularly benefit are those which measure the lifetime of B and D mesons which decay into hadronic final states. The latter, combined with an automated alignment and calibration tasks [4] of the subdetectors in real time using data from HLT1, leads to offline quality information inside the trigger software.

At HLT2 level the $b$ hadrons are selected by inclusive triggers, based on multivariate techniques, which look for a two-, three-, or four-track vertex with significant transverse momentum, significant displacement from the primary vertex and a topology compatible with the decay of $a b$ hadron. For Run II these algorithms have been reoptimized [5] and the relative gain in efficiency with respect to the Run I topological trigger is expected to be between $10 \%$ and $60 \%$ depending on the decay mode. For what concern c hadrons, many exclusive selections have been implemented fully exploiting the offline quality information already available in the trigger. More details are given in the next section. A MVA-based inclusive selection has also been implemented. Inclusive selection is extremely challenging because of the high charm cross section, but it allows to greatly enhance the physics reach, e.g. for decays with neutral particles.

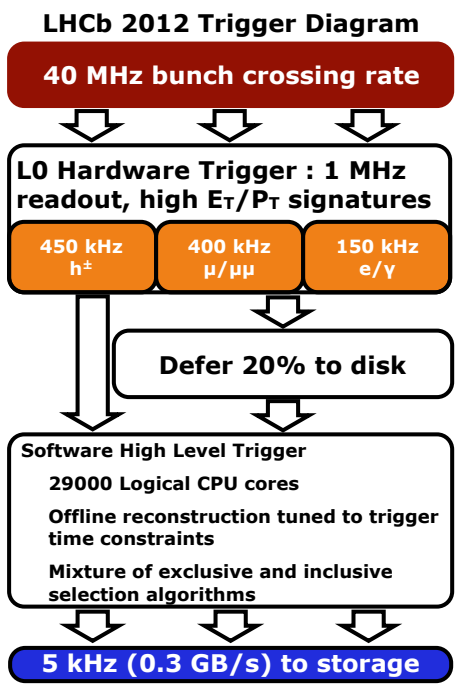

(a)

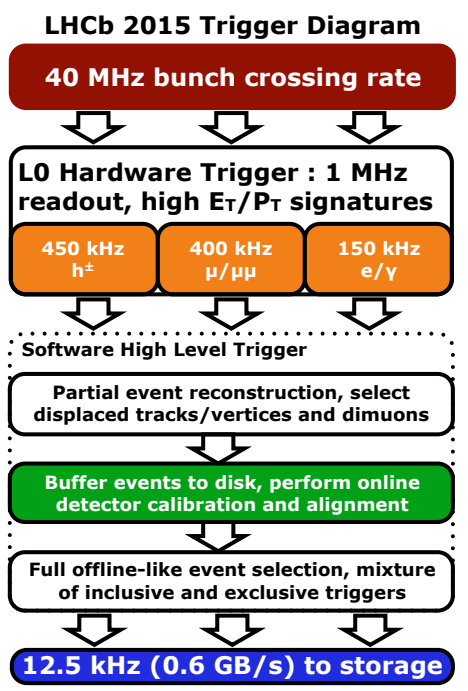

(b)

Figure 1: The LHCb trigger scheme in Run I(a) and in Run II (b) 


\section{Real time analysis}

Offline quality information at the trigger level means that in Run II it is possible to perform physics analyses directly with the information calculated by the HLT event reconstruction using the special stream of data known as Turbo stream [6]. The information calculated by the trigger is used to directly perform physics measurements without the need for further offline reconstruction, saving $\mathrm{CPU}$ and storage resources. Reaching the ultimate precision of the LHCb experiment already in real time as the data arrive has the power to transform the experimental approach to processing large quantities of data. This approach has the advantage that it is possible to save only the information on the signal candidate tracks (about $5 \mathrm{kB}$ per event) instead of all the electronic signals recorded from the detector (about $70 \mathrm{kB}$ per event). This decrease of the event size allows a higher selection rate, which is essential for charm analyses which are dominated by the statistical uncertainty. The whole system has already been tested in 2015 and it has led to two the measurement of the open charm [7] and $J / \psi[8]$ cross section at $\sqrt{s}=13 \mathrm{TeV}$.

\section{Conclusions}

The LHCb trigger has been improved for the Run II data taking. Now it is the first high energy physics experiment implementing a fully automatic tracking system alignment, PID calibration and offline quality event reconstruction in real time. LHCb has completely re-organize its approach to process the huge amount of data coming from proton collisions, with the result of increasing the physics program that it can cover.

\section{References}

[1] LHCb collaboration, The LHCb Detector at the LHC, JINST 3 S08005 (2008).

[2] M. Frank et al., Deferred High Level Trigger in LHCb: A Boost to CPU Resource Utilization, J. Phys. Conf. Ser. 513012006 (2014).

[3] M. W. Kenzie and V. Gligorov, Lifetime unbiased beauty and charm triggers at LHCb, LHCb-PUB-2015-026 (2016).

[4] G. Dujany and B. Storaci, Real-time alignment and calibration of the LHCb Detector in Run II, J. Phys. Conf. Ser. 6648882010 (2015).

[5] Tatiana Likhomanenko et al., LHCb Topological Trigger Reoptimization, J. Phys. Conf. Ser. 6648 082025 (2015).

[6] R. Aaij et al., Tesla : an application for real-time data analysis in High Energy Physics, Comput. Phys. Commun., doi:10.1016/j.cpc.2016.07.022. (2016).

[7] LHCb collaboration, Measurements of prompt charm production cross-sections in pp collisions at $\sqrt{s}=13 \mathrm{TeV}$, ArXiv:1510.01707 (2015)

[8] LHCb collaboration, Measurement of forward $\mathrm{J} / \psi$ production cross-sections in pp collisions at $\sqrt{s}=13 \mathrm{TeV}$, JHEP $10172(2015)$ 